\title{
ON FINITE WEAK AND INJECTIVE DIMENSION
}

\author{
LEO G. CHOUINARD II
}

\begin{abstract}
An extension is given of the theorem relating projective dimension to depth for a finitely generated module of finite projective dimension over a commutative Noetherian local ring. This extension is dualized to relate injective dimension to a concept of codepth when the injective dimension is known to be finite.
\end{abstract}

1. Introduction and notation. A classical result on the projective dimension of modules over a commutative Noetherian local ring says that if the module is finitely generated and of finite projective dimension, then that dimension is the difference between the depth of the ring and that of the module [1, Theorem 3.7]. We extend this result by changing the context to weak dimension. We then dualize to injective dimension, and the result we get seems to be known only in the special case of finitely generated modules.

For the duration of the paper, all rings are commutative Noetherian with unit, and all modules are unitary. If $M$ is an $R$-module, then we denote the weak, projective, and injective dimensions of $M$ over $R$ by w.d. ${ }_{R} M$, p.d. ${ }_{R} M$, and i.d. ${ }_{R} M$ respectively, and we use $E_{R}(M)$ to denote the injective envelope of $M$ over $R$. If $R$ is local with residue field $k$, then we use $M^{v}$ to stand for the Matlis dual $\operatorname{Hom}_{R}\left(M, E_{R}(k)\right)$ of $M$.

If $M$ is any $R$-module, and $I$ is an ideal in $R$, then we define the depth of $M$ over $I$ as the lowest degree in which $\operatorname{Ext}_{R}(R / I, M)$ is nonzero, and the codepth of $M$ over $I$ as the lowest degree in which $\operatorname{Tor}^{R}(R / I, M)$ is nonzero; we denote these by depth $I M$ and codepth $I M$ respectively. If $\operatorname{Ext}_{R}(R / I, M)$ is 0 in all degrees, we say depth $M=\infty$, and likewise for codepth.

We adopt the convention that if we ask for the largest dimension in which a positively graded object is nonzero, and that object is 0 in all degrees, then the requested dimension is $-\infty$. Thus, for example, the projective dimension of 0 over $R$ will be $-\infty$, since it is the supremum over all $R$-modules $A$ of the largest dimension in which $\operatorname{Ext}_{R}(0, A)$ is nonzero.

The author wishes to thank Professor Everett L. Lady for referring him to the article [5] which appears to be the origin of the spectral sequence needed here, and Professor Melvin Hochster for pointing out nonhomological proofs of two of the following corollaries.

2. Depth, dimension, and dualizing.

Proposition 1. If $R$ is a local ring with maximal ideal $m$ and residue field $k$,

Received by the editors December 26, 1975.

AMS (MOS) subject classifications (1970). Primary 13C15, 13D05.

Key words and phrases. Codepth, depth, injective dimension, weak dimension. 
and $M$ is an $R$-module such that w.d. $\cdot_{R} M<\infty$, and $n$ is the largest degree in which $\operatorname{Tor}^{R}(k, M)$ is nonzero, then $n=\operatorname{depth}_{\mathfrak{m}} R-\operatorname{depth}_{\mathfrak{m}} M$.

Proof. By [5], we have a convergent spectral sequence

$$
E_{2}^{p, q}=\operatorname{Tor}_{-p}^{R}\left(\operatorname{Ext}_{R}^{q}(k, R), M\right) \Rightarrow \operatorname{Ext}_{R}^{p+q}(k, M) .
$$

If $n=-\infty$, the spectral sequence is 0 everywhere, so depth $M=\infty$ and the equality holds. Otherwise, the $p=-n, q=\operatorname{depth}_{\mathrm{m}} R$ term is a nonzero corner term of lowest total degree, so it survives and gives the lowest degree in which $\operatorname{Ext}_{R}(k, M)$ is nonzero. This gives $\operatorname{depth}_{\mathfrak{m}} R-n=\operatorname{depth}_{\mathfrak{m}} M$, which transposes to the desired equality.

COROLlaRY 1.1. If in addition to the above assumptions, $M$ is finitely generated, then $p . d ._{R} M=\operatorname{depth}_{\mathfrak{m}} R-\operatorname{depth}_{\mathfrak{m}} M$.

As mentioned earlier, this appeared long ago in [1].

Corollary 1.2. If $R$ is a ring and $M$ is an $R$-module of finite weak dimension, then

$$
w \cdot d \cdot \cdot_{R} M=\sup _{P \text { prime }}\left(\operatorname{depth}_{P}\left(R_{P}\right)-\operatorname{depth}_{P}\left(M_{P}\right)\right) .
$$

Proof. The result follows easily from Proposition 2.2 of [2] and Proposition 1 above.

Proposition 2. If $R$ is a local ring with maximal ideal $\mathrm{m}$ and residue field $k$, and $M$ is an $R$-module such that i.d. ${ }_{R} M<\infty$, and $n$ is the largest degree in which $\operatorname{Ext}_{R}(k, M)$ is nonzero, then $n=\operatorname{depth}_{\mathrm{m}} R-\operatorname{codepth}_{\mathrm{m}} M$.

Proof. This can be proved directly from the other spectral sequence in Ischebeck's article [5]. Instead, however, consider the module $M^{v}$. By Theorem 1.5 of [6], w.d. ${ }_{R} M^{v}=$ i.d. $_{R} M<\infty$. By Propositions VI.5.1 and VI.5.3 of [4],

$$
\operatorname{Ext}_{R}^{m}\left(k, M^{v}\right) \approx \operatorname{Tor}_{m}^{R}(k, M)^{v} \text { and } \operatorname{Ext}_{R}^{m}(k, M)^{v} \approx \operatorname{Tor}_{m}^{R}\left(k, M^{v}\right)
$$

for any degree $m$. Thus codepth $M=\operatorname{depth} M^{v}$, and $n$ is the largest degree in which $\operatorname{Tor}^{R}\left(k, M^{v}\right)$ is nonzero. Thus the proposition follows from Proposition 1 applied to $M^{v}$.

COROLlaRY 2.1. If in addition to the assumptions of Proposition 2 we have that $M$ is finitely generated, then i.d. $\cdot_{R} M=\operatorname{depth}_{\mathrm{m}} R$.

Proof. In these circumstances, Bass [3, Corollary 3.2] has shown that $\mu_{\text {i.d. } M}(\mathfrak{m}, M) \neq 0$. But $n=\sup \left\{j ; \mu_{j}(\mathfrak{m}, M) \neq 0\right\}$ and the result follows.

The above corollary appears as Lemma 3.3 in [3].

Corollary 2.2. $R, k$, and $\mathrm{m}$ as above. Then $\operatorname{depth}_{\mathfrak{m}} R=\operatorname{codepth}_{\mathfrak{m}} E_{R}(k)$.

Proof. Let $M=E_{R}(k)$ in the proposition. Clearly $n=0$.

COROllary 2.3. The map $\mathrm{m}_{R} \otimes_{R}(k) \rightarrow E_{R}(k)$ via multiplication is an epimorphism iff $\operatorname{depth}_{\mathfrak{m}} R \neq 0$, and is an isomorphism iff $\operatorname{depth}_{\mathfrak{m}} R>1$.

Proof. This map embeds in the exact sequence 


$$
0 \rightarrow \operatorname{Tor}_{1}^{R}\left(k, E_{R}(k)\right) \rightarrow \mathfrak{m} \otimes_{R} E_{R}(k) \rightarrow E_{R}(k) \rightarrow \operatorname{Tor}_{0}^{R}\left(k, E_{R}(k)\right) \rightarrow 0 .
$$

The result then follows immediately from Corollary 2.2 and the definition of $\operatorname{codepth}_{\mathrm{m}} E_{R}(k)$.

Professor Hochster has pointed out that the last two corollaries have straightforward proofs by using the identification of $\operatorname{Hom}_{R}\left(E_{R}(k), E_{R}(k)\right)$ with $\hat{R}$, the completion of $R$ at $\mathrm{m}$.

Proposition 3. Let $R$ be a ring, $M$ an $R$-module such that $n=i . d ._{R} M<$ $\infty$, and $I$ an ideal in $R$ maximal with respect to the property that

$$
\operatorname{Ext}_{R}^{n}(R / I, M) \neq 0 .
$$

Then $I$ is prime and $\operatorname{Ext}_{R}^{n}(R / I, M) \approx \operatorname{Ext}_{R}^{n}\left(R / I, M_{I}\right)$.

Proof. If $I$ is not prime, $R / I$ has a tower whose factor modules all look like $R / P_{i}$, where the $P_{i}$ 's are prime ideals which properly contain $I$. But then $\operatorname{Ext}_{R}^{n}\left(R / P_{i}, M\right)=0$ for all $i$, and from the long exact sequence for Ext, $\operatorname{Ext}_{R}^{n}(R / I, M)=0$, a contradiction. Now since $I$ is prime, if $r \in R \backslash I$ we have an exact sequence $0 \rightarrow R / I \rightarrow R / I \rightarrow R /(I, r) \rightarrow 0$ where the first map is multiplication by $r$. Putting this into our long exact sequence for $\operatorname{Ext}_{R}(, M)$, we get

$$
0=\operatorname{Ext}_{R}^{n}(R /(I, r), M) \rightarrow \operatorname{Ext}_{R}^{n}(R / I, M) \rightarrow \operatorname{Ext}_{R}^{n}(R / I, M) \rightarrow 0
$$

where the first term is 0 because of the maximality of $I$. Thus

$$
\operatorname{Ext}_{R}^{n}(R / I, M) \approx \operatorname{Ext}_{R}^{n}(R / I, M)_{I} \approx \operatorname{Ext}_{R}^{n}\left(R / I, M_{I}\right)
$$

COROllary 3.1. If $R$ is a ring and $M$ is an $R$-module of finite injective dimension, then

$$
\begin{aligned}
\text { i.d.R } \cdot_{R} M & =\sup _{P \text { prime }}\left(\operatorname{depth}_{P}\left(R_{P}\right)-\operatorname{codepth}_{P}\left(M_{P}\right)\right) \\
& =\sup _{P \text { prime }}\left(\operatorname{codepth}_{P}\left(E_{R}(R / P)\right)-\operatorname{codepth}_{P}\left(M_{P}\right)\right) .
\end{aligned}
$$

Proof. By Proposition 3 and the fact that i.d. ${ }_{R} M_{P} \leqslant$ i.d..$_{R} M$, it suffices to find the largest dimension in which $\operatorname{Ext}_{R}\left(R / P, M_{P}\right)$ is nonzero as $P$ ranges over all primes. But $\operatorname{Ext}_{R}^{i}\left(R / P, M_{P}\right) \approx \operatorname{Ext}_{R_{P}}^{i}\left((R / P)_{P}, M_{P}\right)$ for all $i$, so by Proposition 2 we have i.d. $R_{R} M=\sup _{P \text { prime }}\left(\operatorname{depth}_{P_{P}}\left(R_{P}\right)-\operatorname{codepth}_{P_{P}}\left(M_{P}\right)\right)$. But depth $P_{P}\left(R_{P}\right)=\operatorname{depth}_{P}\left(R_{P}\right)$, and $\operatorname{codepth}_{P_{P}}\left(M_{P}\right)=\operatorname{codepth}_{P}\left(M_{P}\right)$, giving the first equation. The second comes from Corollary 2.2 and the above relations with $M_{P}$ replaced by $E_{R}(R / P)$.

Note that in the above we have not used the full power of Matlis duality. For example, when $R$ is local and $I$ is any ideal in $R$, it follows from Propositions VI.5.1 and VI.5.3 in [4] that for any $R$-module $M$, $\operatorname{codepth}_{I} M=$ $\operatorname{depth}_{I} M^{v}$ and $\operatorname{depth}_{I} M=\operatorname{codepth}_{I} M^{v}$. Thus since $R^{v}=E_{R}(k)$, Corollary 2.2 is just a special case of the second equality. However, there does not seem to be any direct way to get Corollary 3.1 from Corollary 1.2 since, in general, if $P$ is not the maximal prime, then $\operatorname{codepth}_{P}\left(M_{P}\right)=\operatorname{depth}_{P}\left(M_{P}\right)^{v} \neq$ $\operatorname{depth}_{P}\left(M^{v}\right)_{P}$ when $R$ is local. 


\section{REFERENCES}

1. M. Auslander and D. A. Buchsbaum, Homological dimension in local rings, Trans. Amer. Math. Soc. 85 (1957), 390-405. MR 19, 249.

2. Homological dimension in Noetherian rings. II, Trans. Amer. Math. Soc. 88 (1958), 194-206. MR 20 \#3203.

3. H. Bass, On the ubiquity of Gorenstein rings, Math. Z. 82 (1963), 8-28. MR 27 \#669.

4. H. Cartan and S. Eilenberg, Homological algebra, Princeton Univ. Press, Princeton, N. J., 1956. MR 17, 1040.

5. F. Ischebeck, Eine Dualität zwischen den Funktoren Ext und Tor, J. Algebra 11 (1969), 510-531. MR 38 \# 5894.

6. T. Ishikawa, On injective modules and flat modules, J. Math. Soc. Japan 17 (1965), 291-296. MR 32 \# 5711.

Department of Mathematics, University of Kansas, LaWrence, KanSas 66045

Current address: Department of Mathematics and Statistics, University of Nebraska, Lincoln, Nebraska 68508 